\title{
Consumo recreativo de drogas y reducción de daños en la vida nocturna global
}

\author{
Mark A. Bellis*, Karen Hughes** \\ *Catedrático de Salud Pública, **Adjunto a la Investigación de Salud Pública
}

Enviar correspondencia: M. A. Bellis, Centro de Salud Pública, 70 Great Crosshall Street, Liverpool John Moores University, Liverpool, L3 2AB, Reino Unido. E-mail (para correspondencia) m.a.bellis@livim.ac.uk

\section{Resumen}

El turismo internacional vinculado a la música dance es responsable de que millones de jóvenes viajen al extranjero cada año para disfrutar de la vida nocturna en diferentes países. Debemos considerar tres problemas de salud asociadas a este reciente fenómeno: cambios en las drogas que consumen durante su estancia en el extranjero, variación de los estándares de salud y seguridad de la vida nocturna en un plano internacional, y la situación relativa de los sistemas que suministran información sobre la salud y la vida nocturna a nivel internacional. Las personas que viajan al extranjero continúan extendiendo nuevas tendencias en el consumo de las drogas recreativas. Sin embargo, las redes internacionales para cooperar en la recogida de información de las nuevas tendencias en el consumo de drogas recreativas y en las intervenciones para enfrentarse a las consecuencias asociadas a problemas de la salud, siguen estando muy poco desarrolladas. El desarrollo de la vida nocturna internacional continúa jugando un papel importante en el desarrollo económico de las ciudades y centros de ocio mundiales. No obstante, urge una mayor inversión en el plano internacional que atienda a la reducción de daños, a la información que incumbe a la seguridad y salud, y a la promoción de intervenciones especialmente dirigidas a jóvenes que salen fuera de sus países.

Palabras claves: "Clubbing," drogas recreativas, vida nocturna y salud, violencia, reducción de daños

\section{Summary}

International tourism associated with dance music is now responsible for millions of young people travelling abroad each year to sample nightlife in different countries. We consider three public health issues associated with this recent phenomenon: changes in young people's substance use while abroad, international variations in nightlife health and safety standards and, the status of systems for communicating health and nightlife information on an international basis. Individuals travelling abroad continue to spread new trends in recreational drug use. However, international networks for sharing intelligence on trends in recreational drug use and on interventions to deal with the associated health consequences remain poorly developed. The development of an international nightlife continues to play a vital role in the economic development of cities and resorts worldwide. However, more investment is urgently needed into the international exchange of harm reduction and nightlife health and safety intelligence and into the development of interventions specifically aimed at young people partying abroad.

Key Words: Clubbing," recreational drugs, nightlife health, violence, harm minimisation 


\section{INTRODUCCIÓN}

\section{Transmisión internacional de consumo de droga recreativa}

E n gran parte de Europa, en las dos últimas décadas, se han producido importantes transformaciones en las actividades nocturnas (Chatterton \& Hollands, 2002). Aunque el consumo de alcohol y tabaco siguen siendo los componentes clave de la vida nocturna, se han ido añadiendo cada vez más otras sustancias a la experiencia (Measham et al., 2001). Un factor clave en el aumento del consumo de drogas recreativas es su asociación a clubs nocturnos y música dance (lo que generalmente se conoce como 'clubbing') (Forsyth et al, 1997). El nuevo fenómeno del 'clubbing' ha llegado a muchos países, fundamentalmente desde Ibiza y otros lugares internacionales de esparcimiento, de la mano de personas que pasan sus vacaciones en el extranjero y, cuando regresan, introducen la combinación de música dance y consumo de éxtasis en su país de residencia (Melechi, 1993; Garratt, 1998). Actualmente, se calcula que unos 3,5 millones de personas del Reino Unido acuden a clubs nocturnos cada semana (Mintel International Group Limited, 2002), y las cifras son equivalentes en otros países europeos.

La relación entre el clubbing y el consumo de drogas recreativas queda demostrada con los niveles de consumo de droga entre la población asidua a los clubs nocturnos, en función del tipo de club que prefieren, de la música que eligen y de la frecuencia con que salen (Calafat et al, 2001). Así, en una investigación reciente entre los asistentes a ese tipo de clubs en el Reino Unido, se ha comprobado que el cannabis, el éxtasis y la cocaína alcanzan un nivel de consumo de un $91 \%$, $96 \%$ y $75 \%$, respectivamente (Winstock et al, 2001). Sin embargo, el aumento de la popularidad del clubbing, y su asociación con drogas recreativas, ha contribuido en general al incremento del consumo de drogas entre la población a nivel nacional e internacional.
Por consiguiente, entre los jóvenes de 16 a 24 la experiencia vital de cannabis, éxtasis y cocaína ha aumentado en el Reino Unido desde un $36 \%$, $8 \%$ y $3 \%$, respectivamente, en 1994 , a un $45 \%, 11 \%$ y $10 \%$ en el año 2000 (Ramsay et al, 2001). Estas pautas de comportamiento se repiten también en otros países de Europa (por ej. Noruega, Pedersen \& Skrondal, 1999; Alemania, von Sydow et al, 1002), así como en otros más alejados (EEUU, Rouse et el, 2002). Por otra parte, a pesar de primeras indicaciones que preveían que las drogas recreativas reemplazarían a otras sustancias (por ej. el alcohol), la evidencia señala que se han añadido simplemente al repertorio de sustancias disponibles para el consumo nocturno (Luke, 1999). Así, pues, el alcohol continúa presentando altos niveles de consumo entre los jóvenes en muchos países europeos, y tanto el consumo de alcohol como el de las drogas recreativas sigue incrementándose entre los grupos de gente joven (Hibell et al, 2000).

\section{Una Red Internacional de Clubbing}

Más que cada país tenga tendencias independientes en el consumo de sustancias, sucede que la población amante de la vida nocturna de diferentes países está hoy en día conectada a través de una red internacional de clubs y centros de vida nocturna (véase fig.1). Existen revistas y páginas web que anuncian regularmente las más importanes capitales de "clubbing" del mundo (por ej. Muzik, 2001), y las continuas reducciones en el coste de los viajes internacionales tienen como consecuencia que los viajes de semanas, o incluso de un breve fin de semana, a este tipo de destinos en el extranjero sean cada vez más frecuentes (Mintel International Group Limited, 2000). Este fenómeno, a menudo denominado turismo de música dance (Sellars, 1998), permite que nuevos comportamientos que surgen en un país respecto al consumo de sustancias sean compartidas rápidamente por individuos que, o 
Figura 1. Principales rutas de tráfico de éxtasis y destinos mundiales a los clubs nocturnos

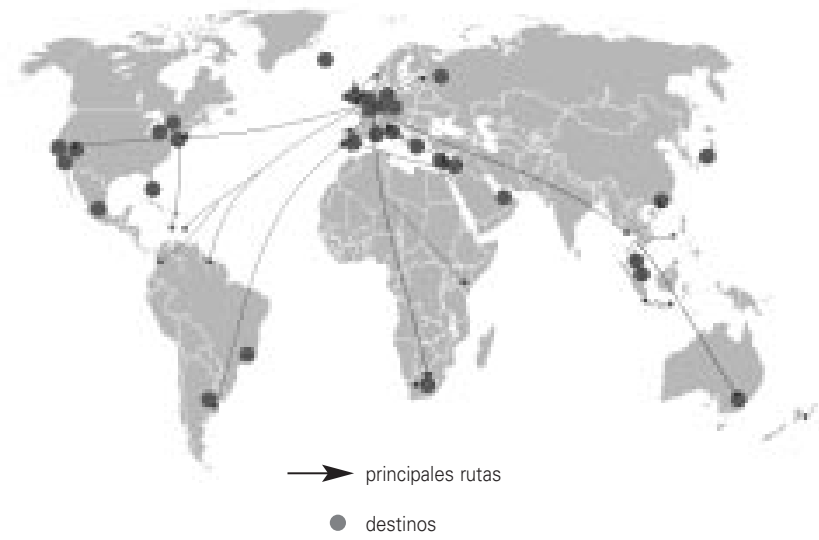

Las principales rutas de tráfico de éxtasis han sido obtenidas del Informe Mundial sobre Drogas 2000 (UNODCCP 2000) y los destinos mundiales a los clubs del Muzik Magazine's Non-Stop Global Clubbing Special (Muzik 2001)

bien han visitado ese país, o se han mezclado con gente del mismo en destinos internacionales de clubbing, tales como Ibiza y Ayia Napa.

Existen una serie de factores que ayudan a la transmisión global de comportamientos recreativos, entre los que se incluye el consumo de drogas.

- Quienes pasan las vacaciones en esa clase de centros internacionales pueden ser líderes en la vida nocturna de sus comunidades locales, sirviendo de modelo e influyendo de un modo desproporcionado en sus amigos y colegas.

- Personajes célebres como los más famosos disc-jockeys trabajan en lugares clave de reunión a nivel internacional, revalorizando la imagen asociada a éstos $y$, por consiguiente, también el atractivo de cualquier comportamiento báquico que puedan representar (por ej. Muzik, 2001).

- Cuando aparecen los centros internacionales de vida nocturna, la industria del viaje fomenta rápidamente paquetes turísticos cerrados, incitando a los jóvenes a visitar tales lugares (Sellars, 1998).
- Algunas compañías anuncian sus productos (desde el alcohol a los coches) asociándolos con destinos populares, utilizando no sólo el nombre de la localidad, sino también ciertas imágenes relacionadas con droga asociadas a ella (Forsyth, 1997).

El éxtasis (como parte de la vida nocturna) ha conseguido penetrar mundialmente en la cultura de la juventud antes incluso de que ese tipo de presiones comerciales y de redes de vida nocturna estuvieran bien establecidas. Hoy en día, con el desarrollo de éstas, el fomento de nuevas drogas (y las tendencias en su consumo) pueden incluso acelerarse. Por supuesto, estos cambios también requieren la expansión de una producción ilícita y la importación de drogas, a lo que las agencias legales se opondrán en la mayoría de los países. No obstante, a pesar de la ayuda que comporta la legislación nacional e internacional, los itinerarios de suministro se adaptan rápidamente a los cambios en la demanda con otras rutas más importantes de suministro del éxtasis, que actualmente conectan entre sí la mayor parte de los centros internacionales de "clubbing" (UNODCCP 2000; Muzik, 2001; Figura 1). 


\section{Desafíos de la Salud Pública}

La globalización de la vida nocturna supone nuevos retos para la salud pública. La frecuencia y duración de las visitas a los lugares de diversión nocturna hacen que una mayor proporción de jóvenes residan regularmente en el extranjero. Sin embargo, poco se conoce acerca del consumo de sustancias legales o ilegales durante las vacaciones y, por lo tanto, de los riesgos de salud que corren estos ciudadanos. Los estándares básicos que regulan la seguridad y salud en el ambiente nocturno varían de unos países y otros, creando riesgos añadidos a los turistas; como es el caso de algunas instalaciones que pueden ser gratuitas en su país de origen (por ej. agua en los clubs) y que resultan caras o inaccesibles en otros lugares (Bellis et al., 2000 a,b). Finalmente, la rápida extensión internacional de nuevas modas en el consumo de drogas puede desembocar en nuevos tipos de comportamientos de riesgo, que pueden llegar a un país antes de que se haya estudiado el mejor modo de abordarlos a nivel internacional. Como consecuencia de esto, analizaremos tres problemas relacionados con la salud pública:
- Los cambios en el consumo de sustancias entre los jóvenes y su comportamiento asociado mientras están fuera de su país.

- Los estándares internacionales para la seguridad y la salud en la vida nocturna.

- La comunicación de la información sobre salud y vida nocturna a nivel internacional.

Consumo de droga entre los jóvenes en el extranjero y comportamiento asociado

A pesar de que ya existen numerosos estudios que identifican la epidemiología del consumo recreativo de drogas, en la mayor parte de los casos éstos describen las pautas de consumo de los individuos en su entorno local (i.e. su lugar de residencia) (por ej, Release, 1997; Riley et el, 2001; Lenton et al, 1997). Sin embargo, son los individuos que eligen visitar los centros internacionales de diversión nocturna los que tienen más probabilidades (comparados con el resto de la población) de ser consumidores de sustancias recreativas, incluso mientras se encuentran en su propio país. (figura 2) (Bellis et el, 2000 a,b). Este hecho sugiere de por sí que los problemas asociados al consumo de drogas, pueden presentarse con más probabilidad entre los que viajan. De cualquier mane-

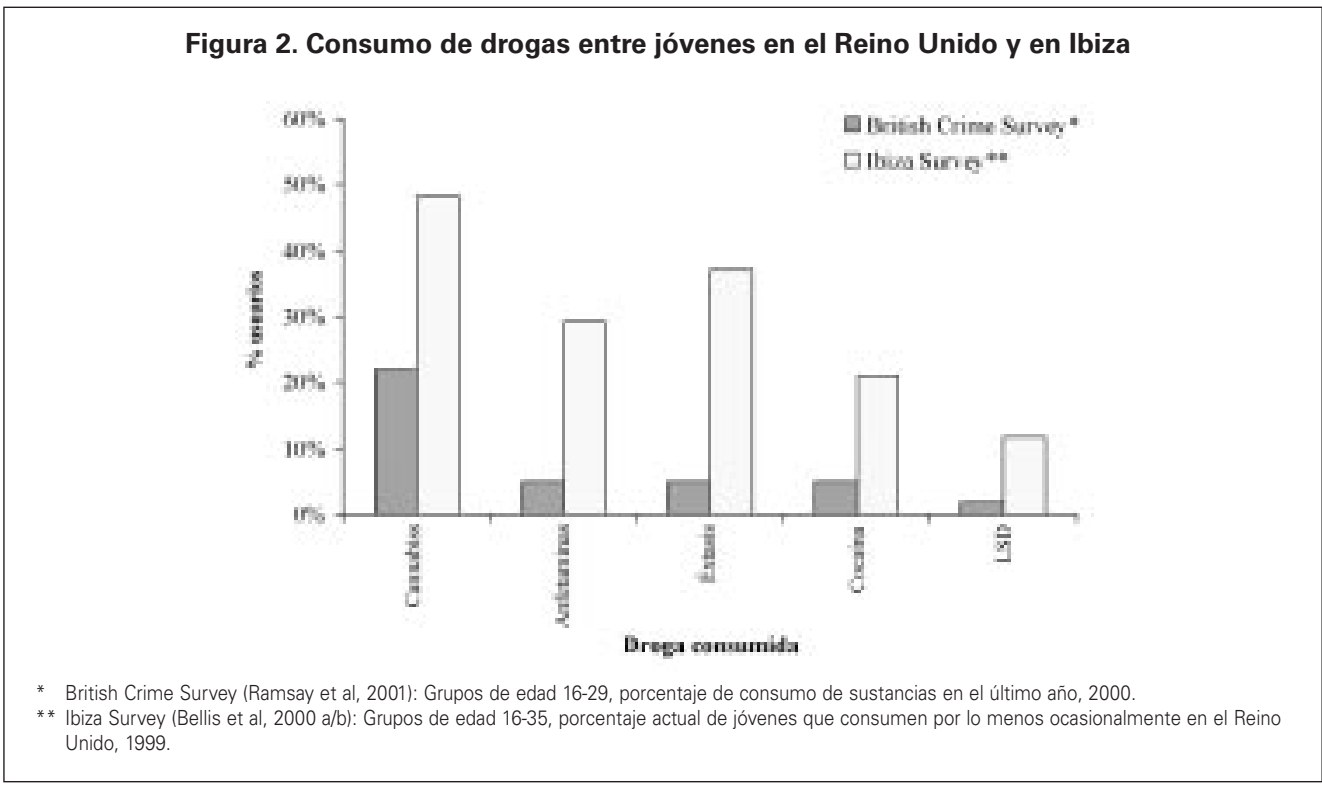


Figura 3. Porcentaje de consumidores de cada sustancia que consumen 5 o más días por semana en el Reino Unido y en Ibiza (individuos que consumen en ambos lugares)

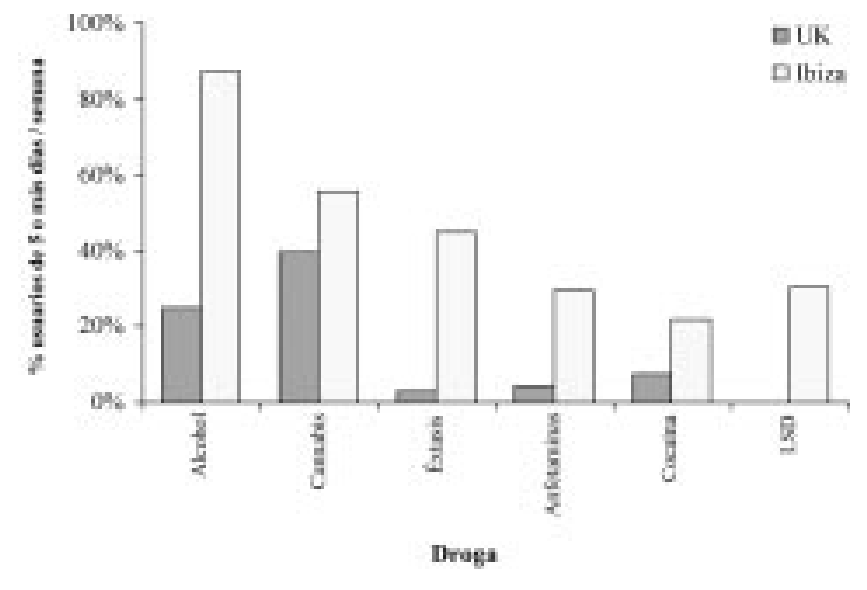

ra, cada vez existen más indicios de que, entre esa población, las vacaciones en el extranjero pueden asociarse al incremento adicional en el consumo de drogas.

Consumo abusivo de drogas en el extranjero como diversión y consecuencias

Los datos recogidos entre numerosos individuos sobre el consumo de drogas en su país, el Reino Unido, y durante su estancia en Ibiza, mostraron cambios sustanciales en el comportamiento exhibido durante las vacaciones. La figura 3 muestra el porcentaje de consumidores de cada sustancia (en ambos lugares) que la consumían cinco o más veces por semana, tanto en el Reino Unido como en Ibiza (Bellis et al, 2000 a,b). Durante las vacaciones, los individuos incrementaron de una manera espectacular el consumo de alcohol y todas las drogas recreativas. De ese modo, mientras en el Reino Unido cada droga se consumía < 1 día por semana (excepto el cannabis, donde el consumo se elevaba a $>=5$ días por semana), en Ibiza y con todas las drogas, el consumo era de 2-4 días, $0>=5$ días por semana. Con respecto a los consumidores de éxtasis, el porcentaje de individuos que la utilizaban $>=5$ días por semana aumentó de un 3.0\% de consumidores en el Reino Unido a un $45.0 \%$ en Ibiza (Bellis et al, 2000 a,b). La media de consumo de éxtasis era, tanto en el Reino Unido como en Ibiza, de unas de 3.5 pastillas por noche. No obstante, debido a la mayor frecuencia del consumo, un usuario medio de éxtasis (entre esta población) ingeriría 4.8 pastillas durante diez días en el Reino Unido, pero 18.1 pastillas durante el mismo periodo en Ibiza (IBIZA Survey 2002 data, Bellis et al, no publicado).

Semejantes cambios en el consumo de drogas no se producen sin consecuencias para la salud. Además, los individuos que visitan estos clubs nocturnos internacionales dance suelen mezclar con regularidad el alcohol con otras drogas recreativas, lo que incrementa el potencial de efectos secundarios negativos (Winstock, 2003). Por otra parte, en los casos en que el daño neurológico se relaciona con la dosis (Reneman et al, 2001), los individuos con experiencias de unas pocas semanas de alto consumo en el extranjero (cada año) de éxtasis (y otras drogas) pueden estar a la larga mucho más expuestos a sufrir lesiones cerebrales que aquellos que consumen una vez por semana en su país de residencia. 
Reclutando nuevos consumidores de droga.

La visita a un nuevo país da la oportunidad de experimentar nuevas drogas. Además, en los centros internacionales de diversión, donde las personas pueden estar ya utilizando estas nuevas drogas, la presión para probarlas puede ser considerable (Apostolopoulos et al, 2002). En consecuencia, de cada 1.000 individuos que visitaron Ibiza, más de 15 consumieron cocaína y más de 30 se lanzaron al consumo de éxtasis, a pesar de no haberlo probado nunca en el Reino Unido. Por otra parte, en relación con otras drogas recientemente aparecidas, como la ketamina y $\mathrm{GHB}$, un gran número de individuos que las utilizaron en Ibiza no las habían consumido nunca en el Reino Unido (Hughes \& Bellis, 2003). A través del reclutamiento de nuevos individuos para el consumo de drogas y del reclutamiento de consumidores habituales para el consumo de nuevas sustancias, los centros internacionales de vida nocturna pueden influenciar en las nuevas tendencias en el consumo de drogas recreativas en diferentes países y continentes. Este tipo de procesos han sido en gran medida responsables del surgimiento del éxtasis como fenómeno global (Melechi, 1993). A pesar de ello, no se ha prestado la menor atención a este fenómeno, ni se ha pensado en utilizar los centros internacionales de vida nocturna como sistemas centinela (de alarma temprana) para detectar nuevas tendencias en el consumo de drogas recreativas en otros lugares.

Las experiencias en el extranjero también conllevan riesgos para la salud. En el año 2001, los medios de comunicación señalaban que cada noche se presentaban en los servicios de urgencia de lbiza al menos dos consumidores noveles de GHB como consecuencia de una sobredosis (BBC, 2002). Sin embargo, muchos otros individuos que ponen en peligro sus vidas pueden fracasar en el intento de acceder a dichos servicios, por su ignorancia de cómo hacerlo en un país que no es el suyo o, en determinados países, porque tales servicios estén poco desarrollados.
La herramienta clave para prevenir estos daños debería de ser el desarrollo de una literatura específica de reducción de daños que identificara los riesgos asociados al consumo de drogas en el extranjero, el peligro añadido que supone el aumento del número de noches en que se consumen, y el hecho de experimentar nuevas drogas en ambientes desconocidos. Este tipo de literatura, especialmente dirigida a los que consumen drogas en el extranjero, se ha empezado a desarrollar recientemente, pero aún está lejos de alcanzar su distribución a nivel mundial (por ej. Health Education Authority, 1999; HIT, 2001).

Las medidas para la minimización de daños pueden reducir, pero no eliminar los riesgos de salud en aquellas personas que continúan bebiendo alcohol de forma abusiva o se encuentran desorientadas por el efecto de las drogas (Shevan et al, 2000). No obstante, también se pueden conseguir en esos casos mejoras importantes, no modificando su comportamiento, sino adaptando el ambiente nocturno a estas situaciones.

\section{Estándares internacionales para la salud y seguridad en la vida nocturna}

\section{Relaciones entre salud y entorno nocturno}

Muchos de los efectos perjudiciales de la vida nocturna para la salud no están relacionados con los efectos directos producidos por la droga sino con el hecho de cómo está diseñado y dirigido el ambiente nocturno (Bellis et al, 2000 a). Así, los informes de muertes producidas por éxtasis se refieren regularmente a la temperatura del entorno la muerte más probable es la producida por insolación. La temperatura del club había alcanzado $40^{\circ} \mathrm{C}$ (Burke, 2001) o, en otros casos, a la falta de instalaciones para contrarrestar los efectos del baile y del consumo de drogas- Una serie de personas se quejó de que había falta de agua (Bowcott, 2001). Fomentar un ambiente propicio y bien preparado significa reconocer que gran número de usuarios de estos clubs consumen regularmente sustancias como el alcohol, drogas y 
tabaco (a menudo combinados) y que esto conlleva, por consiguiente, a distintos efectos desorientadores de carácter fisiológico y psicológico. Por otra parte, el ambiente no sólo afecta a los que acuden al lugar a divertirse sino a aquellos que trabajan o residen cerca de estos establecimientos nocturnos. Así, por ejemplo, la inhalación del humo de tabaco puede producir cáncer entre los empleados del club, incluso si no son fumadores (Johnson et al, 2001), y los jóvenes que residen en ciudades con una intensa vida nocturna o en centros internacionales de vida nocturna consumen generalmente un nivel más elevado de drogas recreativas que los jóvenes de su edad en otros lugares (Laespada \& Salazar, 2002).

Algunas propuestas para una vida nocturna más sana están bien establecidas: asesoramiento en los mensajes para la reducción de daños; surtidores de agua, evitar que se mezcle el alcohol con éxtasis y alternar el consumo con períodos de descanso, facilitar a las personas la información básica para proteger su salud. Sin embargo, entre los distintos países e incluso dentro de cada uno de ellos, existen diferencias notables en la provisión de áreas de refrigeración incorporadas a los clubs, a menudo denominadas áreas de frío (chill out areas), y en el acceso gratis al agua fría. Ello significa que este tipo de actuación para proteger la salud puede ser difícil o imposible de llevar a la práctica en algunos de los centros internacionales de vida nocturna.

Otras medidas básicas se hallan aún lejos de ser reconocidas a nivel internacional. Una pequeña sala bien equipada de primeros auxilios, personal especializado y un fácil acceso a los servicios de emergencia proporcionan la mejor oportunidad para que la población con reacciones adversas a las drogas y el alcohol puedan recuperarse. Cada vez se relaciona más con la violencia el consumo de alcohol y de drogas (especialmente cocaína y metanfetamina). Durante el año 1999, el 19\% del total de actos violentos $(n=3,246,000)$ del
Reino Unido se desarrollaron en el exterior de un pub o club, y, en total, un $40 \%$ de los incidentes violentos se vincularon al consumo del alcohol y un $18 \%$ a la droga (Kershaw et al, 2000). Este tipo de violencia puede reducirse de manera directa colaborando con la policía local y entrenando al personal que vigila la entrada. Aunque la formación de este personal es obligatoria y está muy extendida actualmente en algunos países, en otros es voluntaria y prácticamente inexistente.

Cuando aparece la violencia, los elementos que rodean al consumo del alcohol, tales como las botellas y los vasos, son utilizados a menudo como armas, con el resultado de miles de personas con cicatrices para siempre (Shepherd, 1994). La reducción del número de estos incidentes y la protección de la salud de los asistentes a los clubs puede mejorarse con medidas tales como controlar las botellas y los vasos en los bares y clubs (cuadro 1) y prevenir que la gente los saque a la calle, (Young \& Hirschfield, 1999). Sin embargo, la combinación de botellas en la calle provenientes de los bares y una escasa limpieza de la misma, pueden convertirlas en armas potenciales para aquellos que se inclinan a utilizarlas como tal.

Muchas lesiones nocturnas no son intencionadas. Un lugar de reunión oscuro, sistemas de luces intermitentes y la música a todo volumen se combinan para hacer que el interior de muchos bares y clubs lleve a la desorientación, efecto que se ve incrementado por el consumo de alcohol y otras drogas. En bares y clubs mal diseñados, las consecuencias pueden ser tropezones, caídas y hasta muertes en algunas ocasiones (por ej. Manchester Evening News, 2001)'. Igualmente, en el caso de incidentes graves, como fuego (véase tabla 1), es importante que las salidas estén bien iluminadas, que las puertas contra incendios no estén cerradas con llave, que no se rebasen los límites contra incendios establecidos en los locales, y que el personal de los mismos haya recibido una

(1) Este artículo relata un incidente en el que un joven murió tras caer desde un balcón de una sala de fiestas en Manchester, RU. 
preparación que les cualifique para el control de las masas.

Estas medidas tan sencillas que pueden reducir los terribles efectos de un incendio en un club o en un bar, no se encuentran aún operativas en todo el mundo. De igual manera, estas mismas medidas son ahora fundamentales en clubs que puedan convertirse en el blanco de ataques terroristas o en aquellos donde pueda cundir el pánico entre los clientes al pensar que ése es el caso. ${ }^{2}$

En algunos países, los riesgos de consumir drogas recreativas de una manera inconsciente están aumentando. Una iluminación escasa en el interior de los bares y clubs significa dar una cobertura satisfactoria a las bebidas estimulantes, y las drogas que inducen a la violación (durante una cita) se añaden cada vez con mayor frecuencia a las bebidas de los individuos, con el fin de atontarles y volverlos menos propensos a resistir las presiones para practicar el sexo. Solamente en el Reino Unido, los casos conocidos de "violación por drogas" (implicando drogas como GHB, alcohol o rohypnol; Sturman, 2000) han aumentado de 39 en 1990 a 804 en el año 2000 (The Roofie Foundation, 2001). Sin embargo, es probable que estas cifras sean mucho más elevadas, pues se cree que, en realidad, sólo se denuncia una de cada cinco violaciones (Myhill \& Allen, 2002). La violación propiciada por drogas ha hecho también su aparición en otros países (por ej. Australia; Russo, 2000). No obstante, es el alcohol, con diferencia, la droga que más se consume mientras se practica el sexo (consentido o no) (Strunin \& Hingson, 1992; Sturman, 2000); y es, asimismo, su consumo y el incremento de drogas recreativas en las estancias en el extranjero lo que hace aumentar los niveles de sexo sin protección (Bellis et al, 2000 a,b). En consecuencia, los centros internacionales dance pueden facilitar el intercambio de enfermedades de trans- misión sexual, con incrementos de esta clase de infecciones y de embarazos no deseados después de los períodos de vacaciones (Wellings et al, 1999; Parnell \& Rogers, 1998). Las intervenciones para abordar estos problemas incluyen el propio material para el fomento del deseo sexual, asegurando que pueden adquirirse siempre preservativos (por ej. a través de las máquinas automáticas) y, en caso necesario, tener acceso a una contracepción de emergencia. Se están efectuando esfuerzos considerables para proveer de tales medidas a los jóvenes que frecuentan este tipo de vida nocturna en su país de residencia. Sin embargo, apenas se ha tratado la protección sexual de estas mismas personas en el extranjero, donde pueden desconocer los niveles de las enfermedades de transmisión sexual (por ej, HIV endémica o sífilis), dónde adquirir preservativos o cómo tener acceso a una contracepción de emergencia (Bellis et al, 2002 a).

En general, una gama variada y extensa de iniciativas ambientales, legislativas, etc... definen en la actualidad el buen funcionamiento de cualquier lugar nocturno en un marco saludable (Tabla 1). Sin embargo, muchas son sólo iniciativas locales, poco susceptibles de ser aplicadas globalmente. Con la intención de compartir las ideas de una buena práctica y crear una coherencia a nivel nacional, algunos países han desarrollado una guía de vida nocturna sana (por ej. el Reino Unido, Webster et al, 2002; Nueva Zelanda, Ministerio de Salud, 1999), donde se detallan una serie de medidas que pueden tomarse para mejorar y proteger la salud. Los países que poseen tales normas son más bien excepción, y la mayoría de ellos no han cambiado su legislación para implantarlas. Además, cuantos más individuos viajan al extranjero para disfrutar de la vida nocturna, más necesario se hace mostrar una mayor firmeza, no sólo a nivel nacional sino también en el

\footnotetext{
(2) En febrero del año 2003 murieron veintiuna personas en una sala de fiestas de Chicago, USA, cuando la utilización de un pulverizador de pimienta por parte del personal de seguridad provocó una desbandada. Los clientes declararon que habían creído que el club estaba siendo el blanco de un ataque terrorista (Warren, 2003). A continuación de la bomba colocada en la sala de fiestas de Bali en 2002, se sugirió que los lugares públicos de reunión de gente joven (como los clubs nocturnos) podían ser el blanco de actos terroristas importantes (Lord Carlile, Home Affairs Select Committee, 2003).
} 


\begin{tabular}{|c|c|c|c|c|c|c|}
\hline 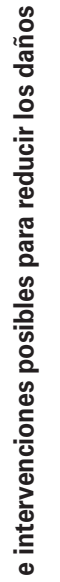 & 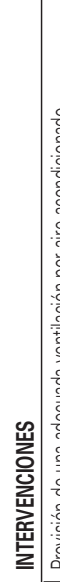 & 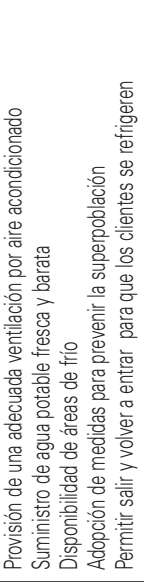 & 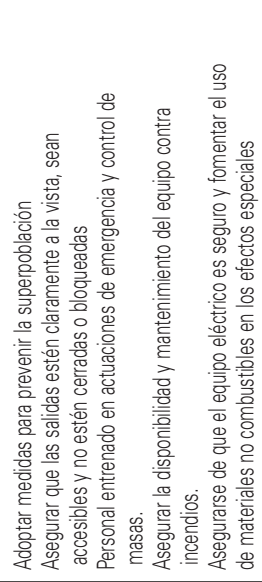 & 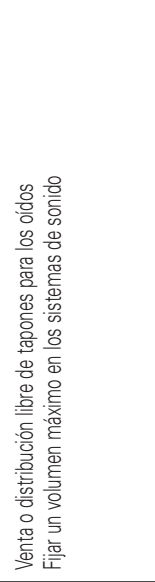 & 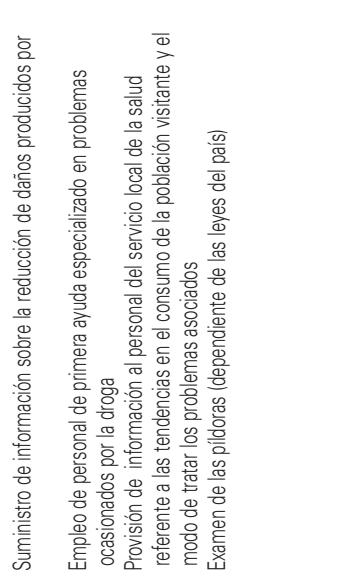 & 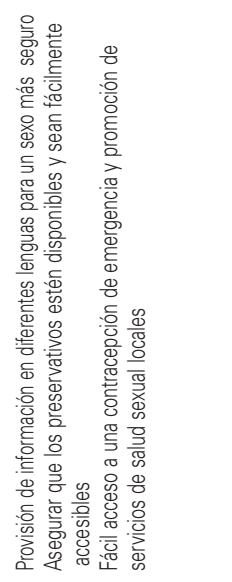 \\
\hline 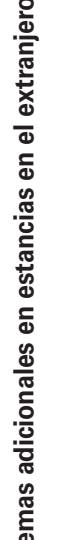 & 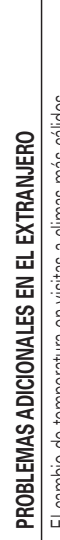 & 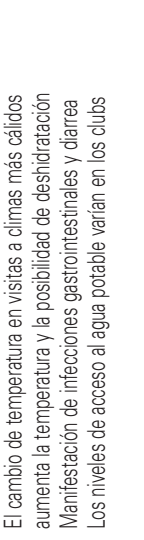 & 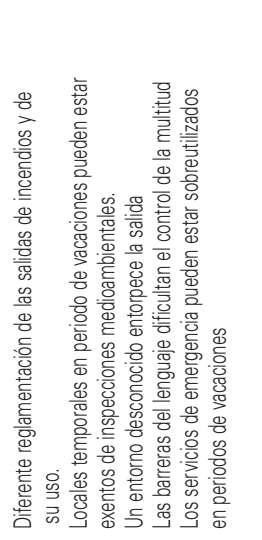 & 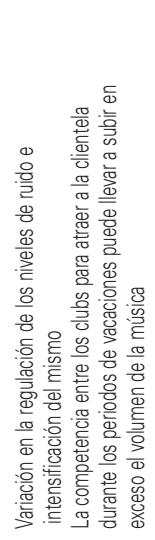 & 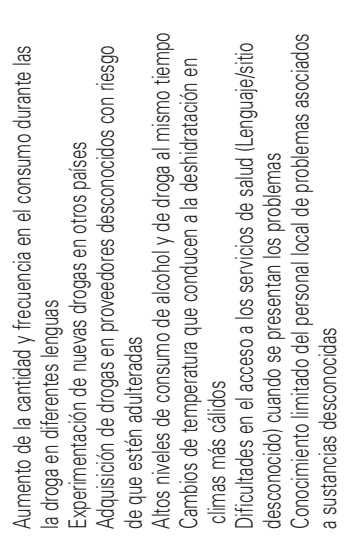 & 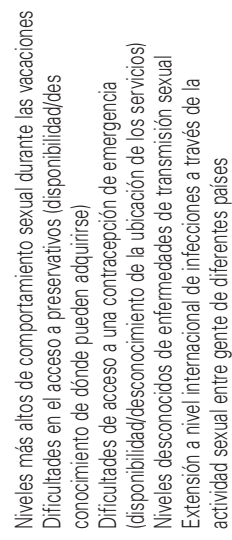 \\
\hline 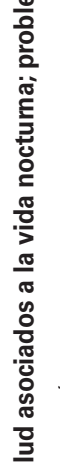 & 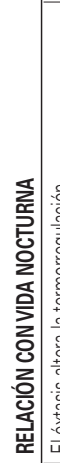 & 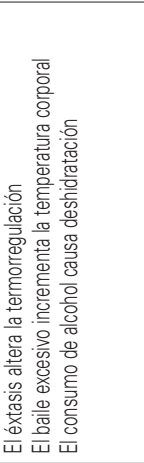 & 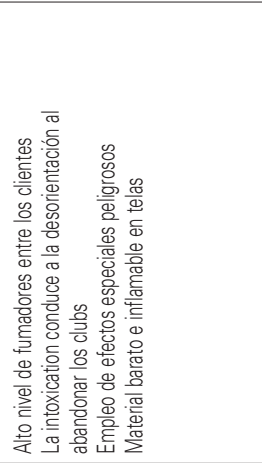 & 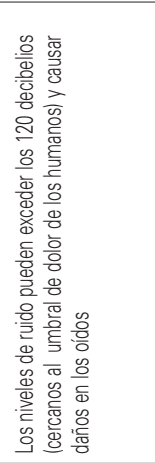 & 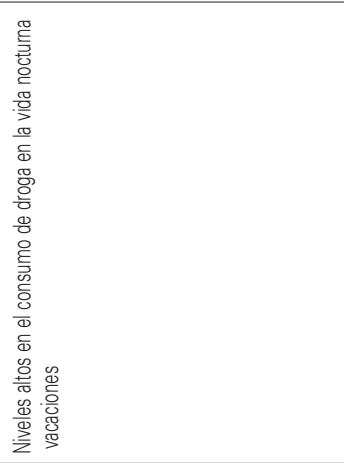 & 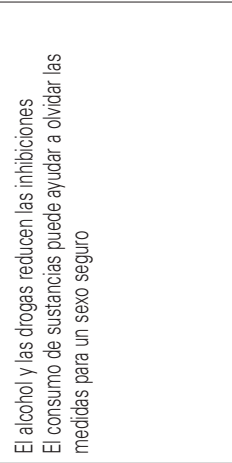 \\
\hline 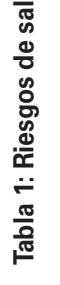 & 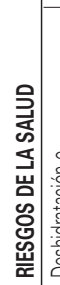 & 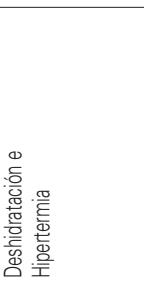 & 裉 & 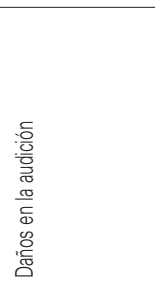 & 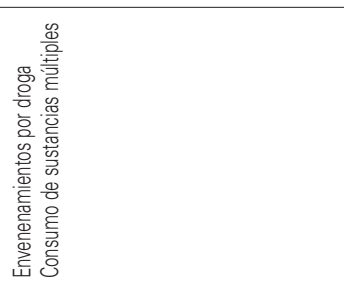 & \\
\hline
\end{tabular}




\begin{tabular}{|c|c|c|c|c|c|}
\hline 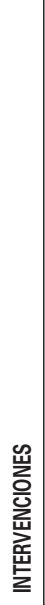 & 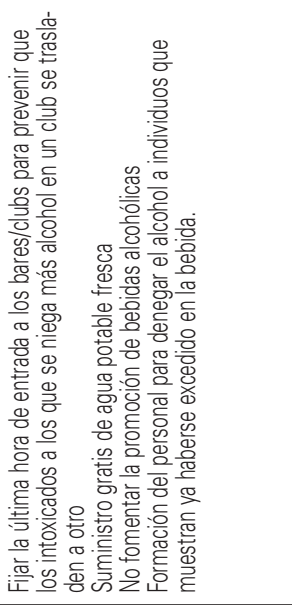 & & 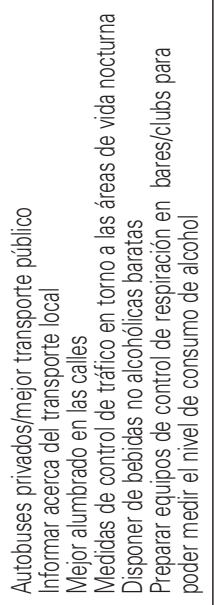 & 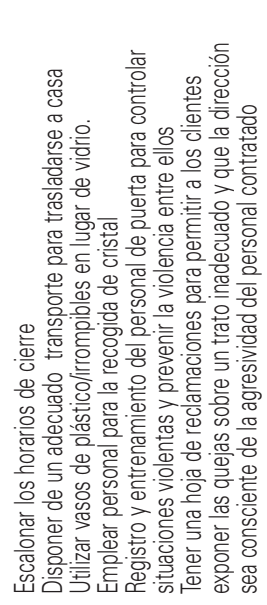 & 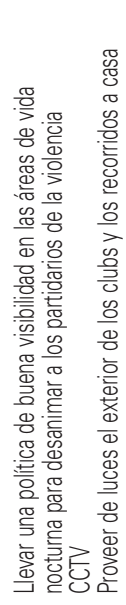 \\
\hline 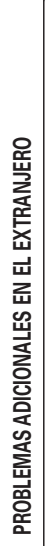 & 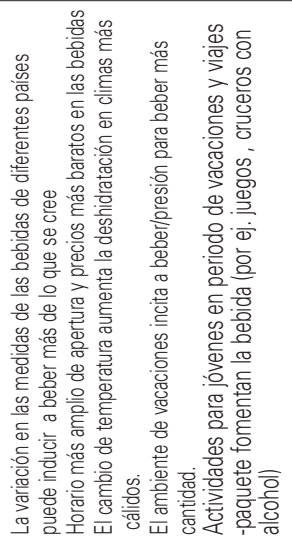 & 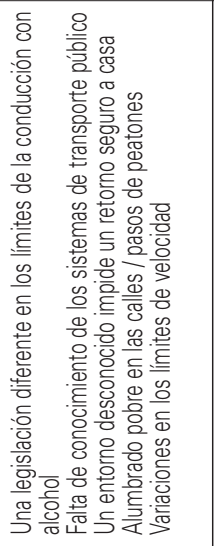 & 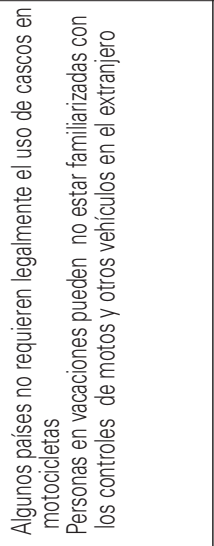 & 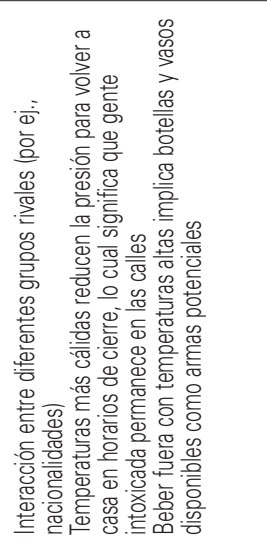 & \\
\hline 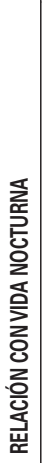 & 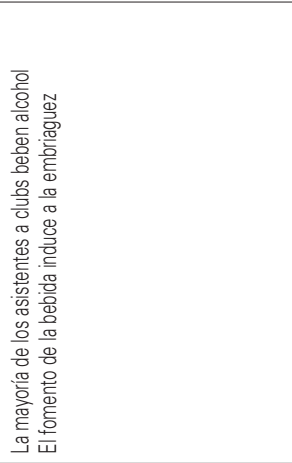 & 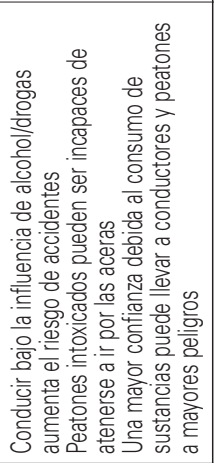 & & 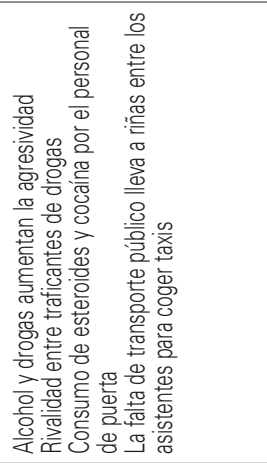 & \\
\hline 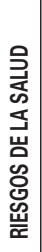 & 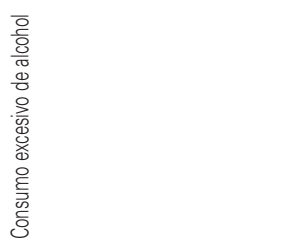 & 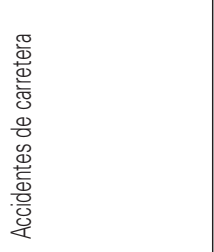 & & $\begin{array}{l}\frac{\pi}{0} \\
\frac{0}{00} \\
\frac{0}{5}\end{array}$ & \\
\hline
\end{tabular}




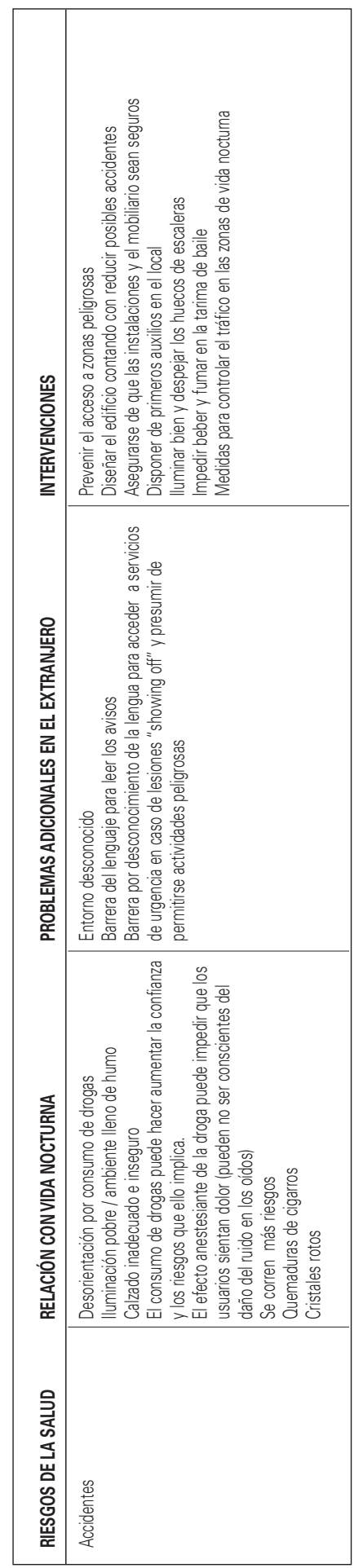

marco internacional. En una breve investigación (Bellis et al, 2000 b) entre personas comprometidas en el desarrollo y en la ejecución de estrategias nocturnas (de diez países diferentes), se les preguntó cuáles eran sus puntos de vista sobre diez áreas de la seguridad nocturna (preparación del personal, tratamiento de las drogas; exceso de calor; locales nocturnos; registro y entrada; información para reducir los daños; primeros auxilios; evacuación del local; control y vigilancia; sucesos en el exterior y lugares de reunión temporales). La mayoría de los consultados estaban de acuerdo en que una serie de medidas básicas, como el adiestramiento del personal para el control de las masas, primeros auxilios y procedimientos de emergencia, o incluso medidas para reducir el exceso de calor (tabla 2) eran muy deseables a nivel internacional. Sin embargo, pocos pensaban que se podrían instaurar a corto plazo. Parece, pues, que no es probable que se produzcan mejoras a corto plazo a través de acuerdos internacionales, sino más bien a través de redes de personas y organizaciones que comuniquen las tendencias en el consumo de drogas recreativas, el desarrollo de la normativa y el impacto de intervenciones relacionadas con la salud.

\section{Comunicar información sobre salud y vida nocturna a nivel internacional}

Proteger la salud de los que se reúnen en los clubs nocturnos, de los que trabajan en ellos o de los que viven cerca de los mismos no es sólo responsabilidad de los servicios de salud. También atañe a la policía, a los servicios ambientales, a las autoridades locales, a las autoridades que conceden las licencias, a los propietarios de bares y clubs, al personal de los mismos y a los usuarios y muchos otros individuos y organizaciones. A nivel local y nacional, esos grupos, trabajando juntos, pueden ayudar a crear entornos que potencien las ventajas económicas y sociales de los lugares nocturnos minimizando los riesgos que implican el consumo de drogas recreativas, el alcohol, la violencia, los accidentes y otras amenazas para la salud. Sin embargo, el éxito de las organizaciones nacionales en el desarrollo de un marco sano en esos centros exige hoy en día información internacional.

Es impresionante el modo en que las nuevas drogas recreativas surgen y se difunden internacionalmente. En algunos países, drogas como la ketamina y GHB han sido absorbidas por la cultura de la vida nocturna (por ej, Australia; Whitten, 2001), mientras otras como la cocaína han 
pasado de ser una rareza a convertirse en algo habitual (por ej. Reino Unido). Otras drogas, como la metamfetamina, ocupan una posición clave en la vida nocturna de algunos países, (por ej. Australia y Tailandia), pero no han penetrado aún de un modo significativo, por ejemplo, en muchos países europeos. Por otra parte, aparecen drogas nuevas en la escena dance en una parte del mundo (por ej.Gamma Butirolactona (GBL) en Australia; Degenhardt, 2003), pero es a través del turismo de la música dance y de los métodos modernos de comunicación (por ej, Internet) cómo conocen sus propiedades, casi inmediatamente, los "clubbers" de todo el mundo (National Drug Intelligence Centre, 2001). La legislación que prohibe el consumo de tales drogas es de aplicación muy lenta y casi siempre resulta relativamente ineficiente. Dejando a un lado la legislación, es imprescindible compartir una rápida información sobre las características de tales sustancias, los riesgos que plantean para la salud y las intervenciones que se han llevado a cabo para intentar reducir sus efectos. Se está desarrollando una gama de iniciativas para intercambiar dicha información. En Europa, por ejemplo, el Consejo de Acción Conjunta para las Nuevas Drogas Sintéticas (Council of Europe's Joint Action on New Synthetic Drugs) ha establecido un sistema de aviso precoz (Early Warning System) para compartir rápidamente la información sobre las propiedades y el riesgo de las nuevas sustancias (Consejo de Europa, 1997). Esa información internacional para tomar parte en las iniciativas y ponerse al corriente de las mismas se ha limitado al consumo de drogas recreativas, con un intercambio de información para una protección mejor y más amplia de la salud en los ambientes nocturnos (alcohol, ruido, violencia, transporte, etc...), estando limitada a redes internacionales y conferencias. Por ejemplo, la iniciativa del Club Health (www.clubhealth.org.uk) aspira a reunir a todas las organizaciones que trabajan en el tema, o que tienen influencia en la vida nocturna local, nacional o internacional, con el fin de intercambiar información sobre la salud en la vida nocturna ( i.e. incluyendo aspectos ambientales, judiciales y de salud). La red Basics (www.basics-network.org) reúne los proyectos para la reducción de daños a través de Europa con idea de intercambiar el conocimiento de los mismos y facilitar el desarrollo de proyectos similares en otras partes del continente europeo. También se observa últimamente un mayor interés a nivel político, y el EMCDDA ha empezado ya a catalogar los tipos de iniciativas que se desarrollan en Europa, especialmente para proteger la salud de los asistentes a bares y clubs (Burkhart \& López, 2002).

Finalmente, añadiremos que también los asistentes a ese tipo de lugares de diversión nocturna tienen que jugar un importante papel en la protección de su propia salud. A lo largo de la última década han surgido una serie de páginas web que no sólo suministran información general dirigida a clubs de un país específico, sino que también detallan los peligros de las drogas utilizadas más comúnmente en esa parte del mundo (www.dancesafe.org; www.ravesafe.org). Aunque van dirigidas principalmente a la población local, también pueden ayudar a los jóvenes que preparan sus viajes al extranjero a evitar algunos de los peligros inherentes al 'clubbing' en dicha localidad. Asimismo, cuando surgen los problemas, aquellos afectados o sus amigos pueden estar mejor informados acerca de las medidas que deben tomar.

\section{Sumario - la situación actual}

Resulta difícil sobreestimar el importante papel que una vida nocturna activa puede desempeñar en el desarrollo económico de ciudades, lugares y centros de vacaciones y en la diversión de millones de seres humanos a lo largo y ancho del mundo. Solamente en un país (Reino Unido), más de 120.000 personas trabajan directamente en la industria de la vida nocturna como empleados de bares y clubs (Oficina de Estadísticas Nacionales, 


\section{Tabla 2: Respuestas recibidas a las preguntas de la Propuesta de Rimini acerca del 'sobrecalenta- miento', porcentaje que aprueba las propuestas y porcentaje que considera las medidas viables}

\begin{tabular}{|c|c|c|}
\hline Propuestas & $\begin{array}{l}\% \text { que está de acuerdo } \\
\text { con la declaración }\end{array}$ & $\begin{array}{l}\% \text { que lo considera } \\
\text { viable }\end{array}$ \\
\hline $\begin{array}{l}\text { Debería existir una zona de frío (chill-out area) donde los usuarios del club pudieran } \\
\text { descansar y refrescarse. Debería estar más fresca y tranquila y tener asientos adecuados }\end{array}$ & $100.0 \%$ & $84.5 \%$ \\
\hline $\begin{array}{l}\text { Debería poder conseguirse libremente en el bar agua fría para beber y no estar restringida } \\
\text { o contaminada en los baños }\end{array}$ & $100.0 \%$ & $74.5 \%$ \\
\hline Debería existir una oferta adecuada de bebidas no alcohólicas en el bar & $100.0 \%$ & $76.6 \%$ \\
\hline $\begin{array}{l}\text { Debería existir una señalización clara y a la vista de los clientes informando donde } \\
\text { se encuentra el agua }\end{array}$ & $97.9 \%$ & $76.1 \%$ \\
\hline $\begin{array}{l}\text { Una adecuada ventilación y aire acondicionado deberían asegurar una temperatura } \\
\text { apropiada y su mantenimiento a lo largo de la velada }\end{array}$ & $97.9 \%$ & $59.6 \%$ \\
\hline Deberían existir guardarropas seguros para permitir a los clientes guardar las prendas adicionales & $89.1 \%$ & $66.6 \%$ \\
\hline $\begin{array}{l}\text { Un sistema adecuado debería facilitar a los clientes el poder quitarse la ropa en caso de } \\
\text { excesivo calor Los empleados deberían tenerlo en cuenta: esto puede indicar una temperatura } \\
\text { más alta de lo recomendable. }\end{array}$ & $95.6 \%$ & $66.7 \%$ \\
\hline Se debería permitir a los clientes volver a entrar en el local si necesitan salir para refrescarse & $95.6 \%$ & $61.4 \%$ \\
\hline $\begin{array}{l}\text { Debería fomentarse que los disc-jockeys pusieran música más lenta de vez en cuando para } \\
\text { reducir el acaloramiento en el baile }\end{array}$ & $61.7 \%$ & $39.1 \%$ \\
\hline $\begin{array}{l}\text { Grandes esfuerzos deberían llevarse a cabo para prevenir el exceso de gente en las zonas } \\
\text { más populares, especialmente en las áreas de enfriamiento }\end{array}$ & $93.6 \%$ & $56.5 \%$ \\
\hline \multicolumn{3}{|c|}{$\begin{array}{l}\text { Las respuestas fueron medidas en una escala de } 1 \text { a } 5 \text { : } \\
\text { Acuerdo con la propuesta: } 1=\text { desacuerdo total, } 2=\text { desacuerdo, } 3=\text { igual o neutral, } 4=\text { de acuerdo, } 5=\text { de total acuerdo } \\
\text { Viabilidad: } 1=\text { no viable en ningún caso, } 2=\text { viabilidad improbable, } 3=\text { neutral, } 4=\text { viabilidad probable, } 5=\text { totalmente viable. } \\
\text { Se han considerado aquellos que han contestado los valores } 405 \text { en cada pregunta } \\
\text { Paises que han participado: Australia, Bélgica, Finlandia, Irlanda, Italia, Países Bajos, Nueva Zelanda, Portugal, Reino Unido y Estados Unidos. }\end{array}$} \\
\hline
\end{tabular}

2002), con miles más trabajando en otras ocupaciones relacionadas con la vida nocturna (por ej. vigilancia en las entradas, disk-jockeys, técnicos de sonido) y en industrias auxiliares, tales como la producción de alcohol, catering e industrias de transporte. Otros se benefician indirectamente, como se vio, por ejemplo, en un estudio que demostró lo importante que era a la hora de elegir universidad el que los jóvenes tuvieran una imagen animada de su población (Young \& Docherty, 2000). Solamente en el Reino Unido, el mercado de los clubs de vida nocturna se ha estimado en 1.8 billones de libras en el 2001 (Mintel International Group Limited, 2002). Los beneficios sociales de la vida nocturna también son importantes. La oportunidad de poder escapar a las presiones de trabajo alivia el estrés (con ayuda del baile), e incluso el ejercicio que se realiza no debería subesti- marse. El 'clubbing' puede también contribuir a crear un sentido comunitario y, a diferencia de otros actos sociales, se ha considerado inclusivo, ya que no tiene en cuenta ni etnias ni clases sociales (Garrat, 1998). Desde hace relativamente poco tiempo, estas comunidades de vida nocturna se han convertido en internacionales, gracias a los viajes baratos y a las modernas tecnologías que conectan dicha vida nocturna entre todos los continentes. Como resultado, la droga recreativa de moda en Australia puede estar mañana en Europa. Lo ideal sería que cuando esas sustancias llegaran a un país, las recomendaciones de cómo enfrentarse a las consecuencias de su consumo para la salud hubieran sido ya compartidas. Sin embargo, esta evolución requiere una mayor comunicación internacional entre profesionales, tanto sobre las nuevas tendencias en el consumo como 


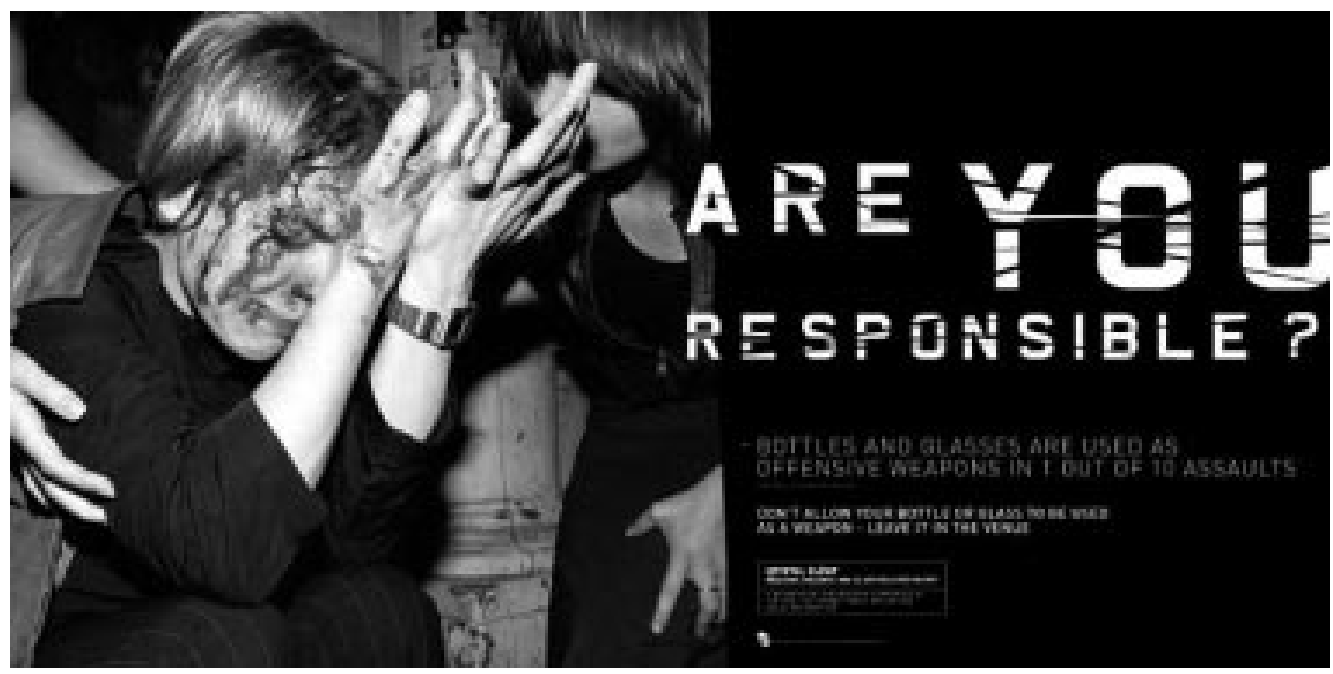

sobre las medidas eficaces para enfrentarse a ellas.

Debemos añadir que el turismo de música dance en clubs nocturnos ha creado sus propias pautas de conducta. Los individuos se comportan de un modo diferente cuando se reúnen fuera de su país. Muchos turistas contemplan cada noche que pasan en el extranjero como una noche de fiesta y consumen todos los días la misma cantidad de droga que tomarían una sola noche a la semana en su propio país. Para proteger la salud de dichos viajeros, son necesarias más intervenciones que se ocupen específicamente de tales comportamientos e identifiquen los peligros de un consumo más frecuente y de la mezcla de diferentes sustancias. Además, las medidas deben reconocer los peligros de adicción planteados por vivir la noche de otros países, donde el clima, la legislación, el acceso a los servicios e incluso los niveles de infecciones (por ej. ETS) pueden diferir de aquellos con los que los individuos están más familiarizados (Tabla 1).

El desarrollo de una vida nocturna internacional continúa ofreciendo grandes oportunidades a la expansión económica y al entendimiento intercultural. Sin embargo, su evolución exige un enfoque más preciso de las naciones sobre los comportamientos recreativos de sus ciudadanos una vez que salen de sus fronteras. Una vida nocturna global significa que el consumo de drogas y los riesgos vinculados a dicho consumo se extienden rápidamente de unos países a otros. Es un desafío para los profesionales de la salud, y otros profesionales, compartir las soluciones a la misma velocidad.

\section{REFERENCIAS}

Apostolopoulos Y, Sonmez S \& Yu GP (2002). HIVrisk behaviours of American spring break vacationers: a case of situational disinhibition? Int $\mathbf{J}$ STD \& AIDS, 13: 733-743.

BBC (2002). Britons import drug problem to Ibiza. http://news.bbc.co.uk/1/hi/programmes/4x4_re ports/2161145.stm, accessed 20th April 2003.

Bellis MA, Hale G, Bennett A, Chaudry M \& Kilfoyle $M$ (2000a). Ibiza uncovered: changes in substance use and sexual behaviour amongst young people visiting an international night-life resort. Int J Drug Policy, 11: 235-244.

Bellis MA, Hale G, Bennett A, Chaudry M \& Kilfoyle M (2000b). Ibiza al descubierto: Cambios en el consumo de drogas y en el comportamiento sexual de los jovenes que pasan sus vacaciones en lugares conocidos internacionalmente por su vida nocturna. Adicciones, 12 (4). 
Bellis MA, Hughes K and Lowey H (2002a). Healthy night clubs and recreational substance use: from a harm minimisation to a healthy settings approach. Addict Behav, 27: 1025-1035.

Bellis MA, Hughes K \& Lowey H (2002b). The Rimini Declaration. Liverpool: Centre for Public Health, Liverpool John Moores University.

Bowcott O (2001). Ecstasy deaths may have been caused by heat, not a bad batch. The Guardian, 30th June 2001.

Burke J (2001). Ecstasy's death toll 'set to go on rising'. The Guardian, 1st July 2001.

Burkhart G \& Lopez ML (2002). Party Setting Projects from EDDRA. Lisbon: EMCDDA.

Calafat A, Fernandez C, Juan M, Bellis MA, Bohrn K, Haddarainen P, Kilfoyle-Carrington M, Kokkevi A, Maalste N, Mendes F, Siamou I, Simon J, Stocco P \& Zavatti P (2001). Risk and control in the recreational drug culture: SONAR project. Palma de Mallorca. IREFREA.

Chatterton P \& Hollands R (2002). Theorising urban playscapes: producing, regulating and consuming youthful nightlife city spaces. Urban Stud, 39: 95-116.

Council of Europe (1997). Joint Action of 16 June 1997 adopted by the Council on the basis of Article K.3 of the Treaty on European Union, concerning the information exchange, risk assessment and the control of new synthetic drugs. Luxembourg: Council of Europe.

Degenhardt L (2003). New Drugs, New Risks. 14th International Conference on the Reduction of Drug Related Harm, Chiang Mai, Thailand, 6-10 April 2003.

Forsyth AJM, Barnard M \& McKeganey NP (1997). Musical preference as an indicator of adolescent drug use. Addiction, 92: 1317-1325.

Forsyth AJM (1997). A quantative exploration of dance drug use: the new pattern of drug use in the 1990s. Thesis, Department of Sociology, University of Glasgow.

Garratt S (1998). Adventures in Wonderland: A Decade of Club Culture. London: Headline Book Publishing.

Health Education Authority (1999). Drugs Abroad: Essential holiday information. London: Health Education Authority.

Hibell B, Andersson B, Ahlstrom S, Balakireva O, Bjarnasson T, Kokkevi A \& Morgan M (2000). The 1999 ESPAD Report: Alcohol and other drug use among students in $\mathbf{3 0}$ European countries. Stockholm: The Swedish Council for Information on Alcohol and Other Drugs (CAN).

HIT (2001). Holiday: Ibiza survival guide. Liverpool: HIT.

Home Affairs Select Committee (2003). Minutes of Evidence for Tuesday 11 March 2003, Lord Carlile of Berriew QC, Reviews of the Government's Terrorism Legislation. HC 515. London: House of Commons.

Hughes K \& Bellis MA (2003). Risk behaviour in Ibiza: results of a four year study. 14th International Conference on the Reduction of Drug Related Harm, Chiang Mai, Thailand, 6-10 April 2003.

Johnson KC, Hu J \& Mao Y (2001). Lifetime residential and workplace exposure to environmental tobacco smoke and lung cancer in never-smoking women, Canada 1994-97. International Journal of Cancer, 93 (6): 902-6.

Kershaw C, Budd T, Kinshott G, Mattinson J, Mayhew P \& Myhill A (2000). The 2000 British Crime Survey. Home Office Statistical Bulletin 18/00. London: Home Office.

Laespada MT \& Salazar L (2002). Oci i temps Iliure. In Joves Balears, Palma de Mallorca: Fundacio Sa Nostra, 150-192.

Lenton S, Boys A \& Norcross K (1997). Raves, drugs and experience: drug use by a sample of people who attend raves in Western Australia. Addiction, 92: 1327-1337.

Luke C (1999). A Little Nightclub Medicine. In Kilfoyle M \& Bellis MA eds. Club Health: The health of the clubbing nation. Department of Public Health, Liverpool John Moores University: Liverpool.

Manchester Evening News (2001). Printworks partygoer dies in 40ft fall. Manchester Evening News. 12th February 2001.

Measham F, Aldridge J \& Parker H (2001). Unstoppable? Dance drug use in the UK clubs scene. In: Parker H, Aldridge J \& Egginton R eds. UK Drugs Unlimited: New research and policy lessons on illicit drug use, 80-97. Basingtoke: Palgrave.

Melechi A (1993). The ecstasy of disappearance. In: Redhead S ed. Rave Off: Politics and deviance in contemporary youth culture, 29-40. Aldershot: Avebury. 
Ministry of Health (1999). Guidelines for Safe Dance Parties - The Big Book. Wellington, New Zealand: Ministry of Health.

Mintel International Group Limited (2000). Youth Holidays. London: Mintel International Group Limited.

Mintel International Group Limited (2002) Nightclubs. London: Mintel International Group Limited.

Muzik (2001). Your passport to Worldwide debauchery. Muzik, no.74, July 2001.

Myhill A \& Allen J (2002). Rape and sexual assault of women: the extent and nature of the problem. Findings from the British Crime Survey. Home Office Research Study 237. London: Home Office.

National Drug Intelligence Center (1999). Drugs and the Internet: An overview of the threat to America's Youth. Johnstown, USA: National Drug Intelligence Center.

Office for National Statistics (2002). Labour Force Survey, March-May 2002. London: Office for National Statistics.

Parnell AM \& Rodgers JL (1998). Seasonality of induced abortion in North Carolina. J Biosoc Sci, 30: 321-332.

Pederson W \& Skrondal A (1999). Ecstasy and new patterns of drug use: a normal population study. Addiction, 94: 1695-1706.

Ramsay M, Baker P, Goulden C, Sharp C \& Sondhi A (2001). Drug misuse declared in 2000: results from the British Crime Survey. London: Home Office.

Release (1997). Drugs, \& Dance Survey: An insight into the culture. London: Release.

Reneman L, Lavalaye J, Schmand B, de Wolff FA, van den Brink W, den Heeten GJ \& Booij J (2001). Cortical serotonin transporter density and verbal memory in individuals who stopped using 3, 4-Methylenedioxymethamphetamine (MDMA or "ecstasy"). Arch Gen Psychiatry, 58: 901-906.

Riley SCE, James C, Gregory D, Dingle H \& Cadger $M$ (2001). Patterns of recreational drug use at dance events in Edinburgh, Scotland. Addiction, 96: 1035-1047.

Rouse B, Sanderson C \& Feldmann J (2002). Results from the 2001 National Household Survey on Drug Abuse: Volume I. Summary of National Findings. Rockville: Substance
Abuse and Mental Health Services Administration, Office of Applied Studies.

Russo L (2000). Date Rape: A hidden crime.

Trends and Issues in Crime and Criminal Justice, 157. Australian Institute of Criminology.

Sellars A (1998). The influence of dance music on the UK youth tourism market. Tourism Manag, 19 (6): 611-615.

Shepherd J (1994). Preventing injuries from bar glasses. Br Med J, 308: 932-933.

Shewan D, Dalgarno P \& Reith G (2000). Perceived risk and risk reduction among ecstasy users: the role of drug, set, and setting. Int J Drug Policy, 10: 431-453.

Strunin L \& Hingson R (1992). Alcohol, drugs, and adolescent sexual behavior. The Int J Addict, 27 (2): 129-146.

Sturman P (2000). Drug Assisted Sexual Assault. London: Home Office.

The Roofie Foundation (2001). Statistical Update: April 2001. http://www.roofie.org.uk, accessed 20th April 2003

United Nations Office for Drug Control and Crime Prevention (2000). World Drug Report 2000. Oxford: Oxford University Press.

von Sydow K, Lieb R, Pfister H, Hofler M \& Wittchen HU (2002). Use, abuse and dependence of ecstasy and related drugs in adolescents and young adults - a transient phenomenon? Results from a longitudinal community study. Drug Alcohol Depend, 66: 147-159.

Warren M (2003). 21 killed in Chicago nightclub stampede. The Telegraph, 18th February 2003.

Webster R, Goodman M \&Whalley G (2002). Safer Clubbing: guidance for licensing authorities, club managers and promoters. London: Home Office.

Wellings K, Macdowell W, Catchpole M \& Goodrich $J$ (1999). Seasonal variations in sexual activity and their implications for sexual health promotion. J R Soc Med, 92: 60-64.

Whitten $L$ (2001). Conference highlights increasing GHB abuse. NIDA Notes 16, 10-11.

Winstock AR, Griffiths P \& Stewart D (2001). Drugs and the dance music scene: a survey of current drug use patterns among a sample of dance music enthusiasts in the UK. Drug Alcohol Depend, 64: 9-17. 
Winstock A (2003). The problems of poly-drug misuse. 14th International Conference on the Reduction of Drug Related Harm, Chiang Mai, Thailand, 6-10 April 2003.

Young C \& Hirschfield A (1999). Crystal Clear Reducing Glass Related Injury An evaluation conducted on behalf of the Safer Merseyside Partnership. Liverpool: University of Liverpool.
Young Z \& Docherty T (2000). Changing Expectations? North West undergraduates' perceptions and expectations of the graduate labour market. Manchester: University of Manchester. 
